EUROPEAN ORGANIZATION FOR NUCLEAR RESEARCH

CERN - AB Division

CERN-AB-2003-014 ABP

\title{
Coherent tune shifts measured with few bunches in the SPS and comparison with resistive wall theory
}

\author{
H. Burkhardt, A. Koschik, G. Rumolo, F. Zimmermann, B. Zotter
}

\begin{abstract}
Coherent tune shifts with current have been measured in the SPS with single bunches and two bunches at varying distances between them. The measurements showed the usual negative slope with intensity in the vertical plane and a small positive one in the horizontal direction. The results are compared with theoretical predictions which include estimates of tune shifts due to the impedance of resistive vacuum chambers with non-circular geometry and finite wall thickness.
\end{abstract}

Presented at PAC 2003, Portland, Oregon, USA

from 12 to 16 May 2003

Geneva, Switzerland

22 May 2003 


\section{Coherent tune shifts measured with few bunches in the SPS and comparison with resistive wall theory}

\begin{tabular}{lccc} 
H. Burkhardt, A. Koschik, G. Rumolo, F. Zimmermann, B. Zotter, CERN, Geneva, Switzerland \\
Abstract & \multicolumn{3}{c}{ Table 1: Relevant SPS parameters } \\
\cline { 2 - 5 } \begin{tabular}{lcc} 
Coherent tune shifts with current have been measured \\
\cline { 2 - 4 } in the SPS with single bunches and two bunches at vary-
\end{tabular} & variable & symbol & value \\
ing distances between them. The measurements showed & revolution frequency & $f_{\text {rev }}$ & $26 \mathrm{GeV/c}$ \\
the usual negative slope with intensity in the vertical plane & & $43347 \mathrm{~Hz}$ \\
and a small positive one in the horizontal direction. The & betatron tunes & $Q_{x, y}$ & $23.07 \mu \mathrm{s}$ \\
results are compared with theoretical predictions which in- & synchr. tune (at 2 MV) & $Q_{s}$ & $5.6 \times 10^{-3}$ \\
clude estimates of tune shifts due to the impedance of re- & momentum compaction & $\alpha_{c}$ & $1.86 \times 10^{-3}$ \\
sistive vacuum chambers with non-circular geometry and & bunch population & $N$ & $(1-12) \times 10^{10}$ \\
finite wall thickness. & rms bunch length & $\sigma_{t}$ & $\sim 0.7 \mathrm{~ns}$ \\
\cline { 2 - 4 } & & &
\end{tabular}

\section{INTRODUCTION}

Coherent tune shifts with current have been measured precisely on single bunches in the SPS for several years $[1,2]$ This allowed to monitor the decrease of the SPS impedance by hardware improvements [3].

The usual negative slope with intensity, corresponding to a defocusing effect of the wake fields is observed in the vertical plane. In the horizontal plane instead, a very small positive slope is observed. Most of the SPS beam pipe consists of flat chambers with horizontal to vertical aspect ratios of $2.5-4$. This asymmetry results in quadrupolar forces. They approximately cancel the focusing effect of the dipole wake fields in the horizontal plane and increase instead the detuning with intensity in the vertical plane [4].

We were motivated to extend the SPS studies to more than one bunch by observations in other machines (PEP2), in which positive slopes have only been observed with multiple bunches. The measurements were done on single bunches and two bunches at various distances. The restriction to very few bunches avoids any complication by electron cloud effects present for many bunches.

\section{MEASUREMENTS}

The measurements were performed in summer 2002, with a single bunch or two bunches of protons injected into the SPS. The relevant beam parameters are summarized in Table 1 .

The SPS was operated in a special $20.7 \mathrm{~s}$ long cycle with a $10.86 \mathrm{~s}$ long injection plateau. The first bunch was injected at the beginning of the cycle and its intensity varied by scraping in the vertical plane at $1.5 \mathrm{~s}$. The second bunch (if present), was injected at $9.6 \mathrm{~s}$. The delay between the two bunches around the ring was chosen by rf-bucket selection. For the main measurements presented here, the distance between the two bunches was selected to be half a turn (about $12 \mu \mathrm{s}$ ). Additional measurements in which the second bunch preceded the first by 2 and $4 \mu$ s were also performed.
The tune measurements were done on the first bunch towards the end of the injection plateau, at $10 \mathrm{~s}$ from the cycle start (after the injection of the second bunch). Bunch lengths were monitored using a longitudinal pick-up and a digital scope. The bunch length was observed to increase slightly with intensity, from $\sigma_{t}=0.65 \mathrm{~ns}$ at the lowest to $0.83 \mathrm{~ns}$ at the highest intensities. Figures 1,2 show the results in terms of the coherent tune shift with intensity, normalized to $\sigma_{t}=0.5 \mathrm{~ns}$. The slope of the curves in the horizontal and vertical plane is a measure of the effective transverse impedance in these planes.

Table 2: Coherent tune shift results, normalized to $0.5 \mathrm{~ns}$ bunch length.

\begin{tabular}{|c|c|c|}
\hline & $\Delta Q_{x} / \Delta N_{p}\left[10^{10}\right]$ & $\Delta Q_{y} / \Delta N_{p}\left[10^{10}\right]$ \\
\hline single & $(+1.1 \pm 0.3) \times 10^{-4}$ & $(-24.2 \pm 0.4) \times 10^{-4}$ \\
two & $(+1.1 \pm 0.2) \times 10^{-4}$ & $(-24.8 \pm 0.6) \times 10^{-4}$ \\
\hline
\end{tabular}

The results obtained by straight line fits are shown in the figures and summarized in Table 2. The uncertainties quoted are obtained from the scattering of the data points around the fitted lines.

No significant difference between the single and two bunch case was observed. This was still the case for 2 and $4 \mu$ s delay between the bunches.

\section{PREDICTIONS}

The coherent transverse tune shift $\Delta \Omega$ due to the finite resistivity of a circular symmetric vacuum chamber wall can be expressed by the functions $g(q)+i f(q)$,

$$
\Delta \Omega_{c o h .} \propto g(q)+i f(q),
$$

where $q$ is the non-integer part of the betatron tune in either of the transverse planes $x$ or $y$. These functions are defined as an infinite sum over all previous revolutions $k$, and in 


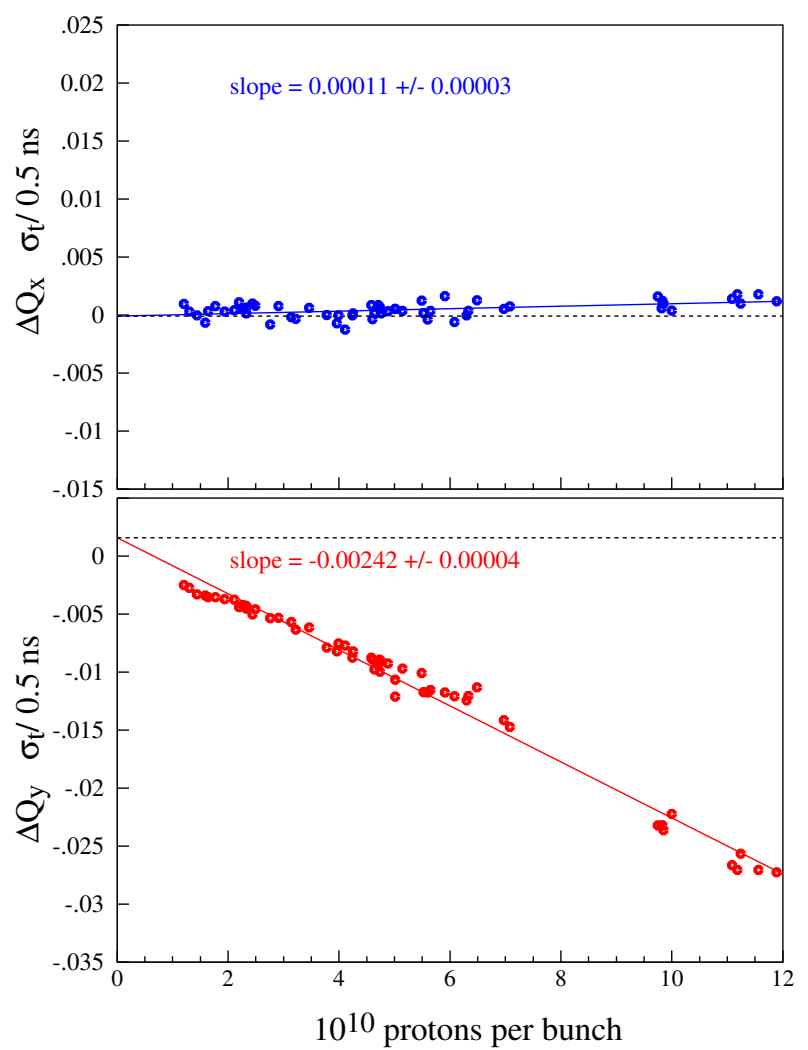

Figure 1: Coherent tune shift with intensity measured for a single bunch in the SPS.

the case of multiple bunches as an additional sum over all bunches,

$$
g(q)+i f(q)=\frac{1}{n_{b}} \sum_{m=0}^{n_{b}-1} \sum_{k=1}^{\infty} \sqrt{\frac{2}{k^{\prime}}} \exp \left(2 \pi i q k^{\prime}\right),
$$

with

$$
\begin{aligned}
k^{\prime} & =k+m \cdot S_{b} / C \\
n_{b} & \ldots \text { number of bunches } \\
0 \leq m<n_{b} & \ldots \text { coupled bunch mode number } \\
S_{b} & \ldots \text { bunch spacing } \\
C=2 \pi R & \ldots \text { circumference. }
\end{aligned}
$$

The single bunch case (see e.g. [5, p.177]) is included by setting $n_{b}=1$.

In non-circular symmetric vacuum chambers additional quadrupolar wakes must be added [6,7], which are proportional to the offset of the test bunch itself

$$
\begin{aligned}
& g(q)+i f(q)_{ \pm \ldots x / y \text { plane }}= \\
& \frac{1}{n_{b}} \sum_{m=0}^{n_{b}-1} \sum_{k=1}^{\infty}\left[\sqrt{\frac{2}{k^{\prime}}} \exp \left(2 \pi i q k^{\prime}\right) \pm \sqrt{\frac{2}{k^{\prime}}}\right] .
\end{aligned}
$$

In the circular symmetric case both horizontal and vertical tune shifts are of equal (negative) size. The additional

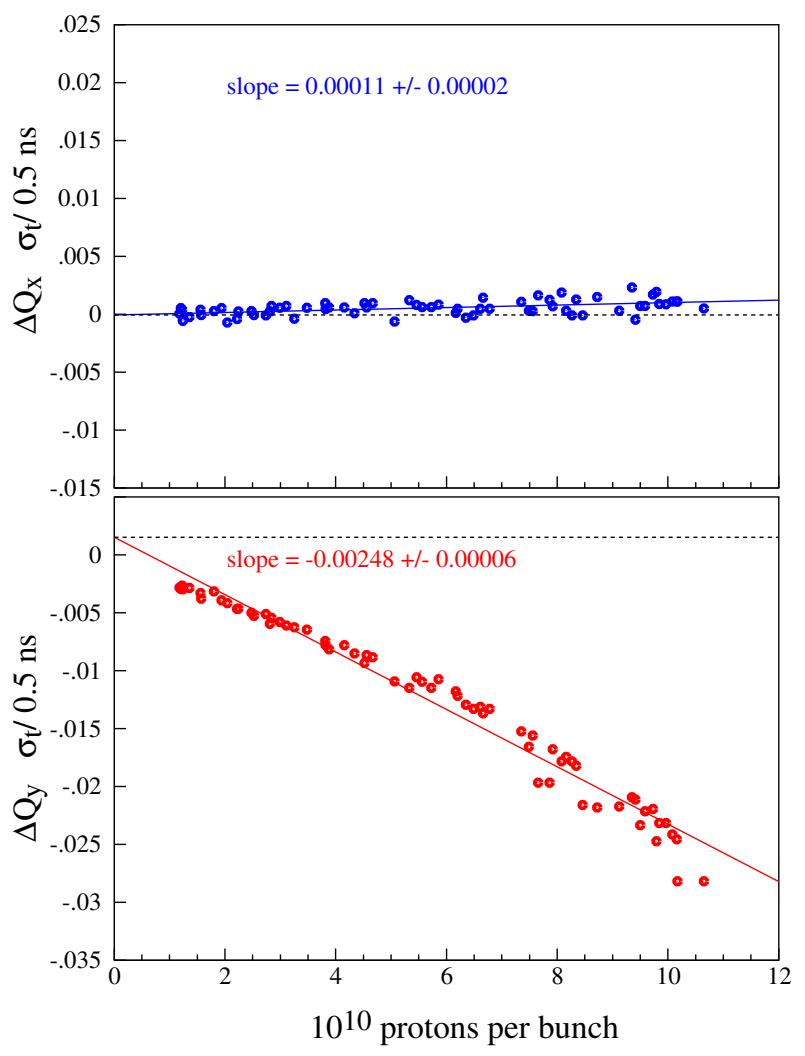

Figure 2: Coherent tune shift with intensity measured on the first of two bunches spaced by half a turn $(12 \mu \mathrm{sec})$.

term $h= \pm \frac{1}{n_{b}} \sum \sum \sqrt{\frac{2}{k^{\prime}}}$ appears only for non-circular symmetric chambers. It reduces or even compensates the horizontal tune shift, while it further increases the vertical one.

The infinite sum $h$ diverges, but a natural truncation occurs when the electromagnetic fields penetrate the chamber wall of finite thickness $t$. In that case the wake function transforms into an exponentially decaying one [8], hence $h$ becomes

$h= \pm \frac{1}{n_{b}} \sum_{m=0}^{n_{b}-1}\left(\sum_{k=1}^{\hat{k}} \sqrt{\frac{2}{k^{\prime}}}+\sqrt{\frac{2}{\hat{k}^{\prime}}} \sum_{k=\hat{k}+1}^{\infty} \exp \left(-\alpha k^{\prime}\right)\right)$,

where $\alpha=\pi \delta_{0} /(\kappa b t), \delta_{0}$ the skin depth at revolution frequency $\omega_{0}, b$ the vacuum chamber radius, and

$$
\kappa=1+b^{2} / d^{2}
$$

for a perfect magnet at radius $d$ [8]. The "cut-off turn number" $\hat{k}$ can be determined approximately by equating the square-root and the exponential wakes,

$$
\hat{k} \approx t^{2} /\left(\pi \delta_{0}\right)^{2}
$$

In the SPS, where $\delta_{0} \approx t \longrightarrow \hat{k} \approx 1 / \pi^{2}<1$, therefore the summation over $k$ starts immediately with the exponential terms. The infinite sum can now be evaluated analytically as geometric series, and we obtain for a single bunch 


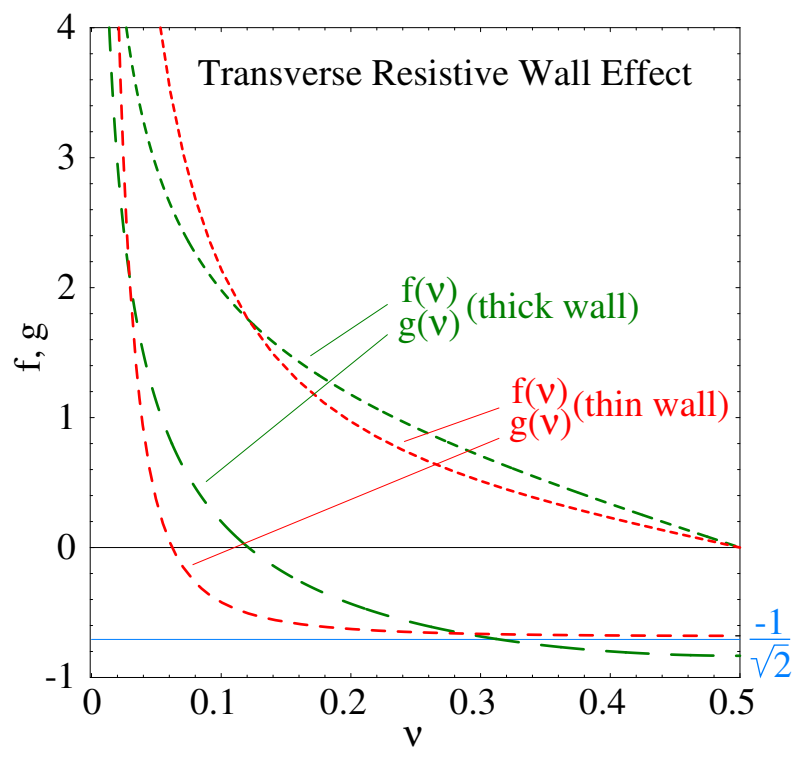

Figure 3: The functions $g(q)$ and $f(q)$ of Eq.(2) and (7). The thin wall graph is drawn for SPS parameters $\left(\delta_{0}=t=\right.$ $2 \mathrm{~mm}, b=20 \mathrm{~mm})$

$$
\begin{aligned}
\left(n_{b}=1 \text { and } k^{\prime}=k\right) & : \\
g(q)+i f(q) & =\sqrt{\frac{2}{\hat{k}}} \sum_{k=1}^{\infty} \exp (-\alpha k+2 \pi i q k) \\
& =\sqrt{\frac{2}{\hat{k}}} \frac{1}{\exp (\alpha-2 \pi i q)-1}
\end{aligned}
$$

For very small $\alpha \ll 1$, the real part of the infinite sum becomes approximately $-1 / \sqrt{2}$, as can be seen from Fig.(3). Furthermore one finds

$$
g(q)=-1 /(\sqrt{2 \hat{k}}) \approx \pi \delta_{0} /(\sqrt{2} t),
$$

independent of the tune. However, for $q$ close to zero this approximation breaks down and one has to use the exact expression to obtain

$$
h=g(0)=\frac{\sqrt{2}}{\sqrt{\hat{k}}\left[\mathrm{e}^{\alpha}-1\right]} \approx \sqrt{2} \frac{\kappa b}{\delta_{0}},
$$

where the last approximation is valid for $\alpha \ll 1$.

For the SPS, we get $g \approx-2.2$, while $h \approx 28.3$ is the dominant term. Hence the horizontal tune shift for a single bunch is close to zero or even slightly positive as already reported in [8].

\section{CONCLUSION}

We have measured coherent tune shifts with current in the SPS. The usual negative slope is found in the vertical plane and a small positive slope in the horizontal plane. A much smaller slope in the horizontal plane is expected for a flat chamber geometry, in which quadrupolar wake fields enhance the vertical tune shift and largely cancel the horizontal tune shift.

Positive slopes have already been seen for bunch trains in PEP-II [8] and have been explained in terms of long range resistive quadrupolar wakes which add up coherently over successive bunches and several turns.

In the SPS, we already observe a small positive slope in the horizontal plane for a single bunch and did not see a significant change in case of the presence of a second bunch.

A reduced multi-bunch effect in the SPS compared to PEP-II is in fact expected due to the larger skin depth at revolution frequency in the stainless steel vacuum chamber and the larger SPS-circumference. For the SPS, the wake function changes from the usual inverse square-root dependence on distance to an exponentially decaying one in less than one turn.

\section{REFERENCES}

[1] H. Burkhardt, G. Rumolo, and F. Zimmermann, "Measurements of SPS Single-Bunch Coherent Tune Shifts and Head-Tail Growth Rates in the Year 2001", CERN, SL Note 2001-043 (MD), 7-12-2001.

[2] H. Burkhardt, G. Rumolo, and F. Zimmermann, "Coherent beam oscillations and transverse impedance in the SPS", Proc. EPAC 2002 pp. 1449 - 1451 and CERN-SL-2002-030.

[3] P. Collier, M. Ainoux, et al., "Reducing the SPS Machine Impedance", Proc. EPAC 2002 pp. 1458 - 1460 and CERN-SL-2002-026-MR.

[4] J. Gareyte, "Impedances: Measurements and Calculations for Non-symmetric Structures", Proc. EPAC 2002 pp. 89 93 and CERN-SL-2002-028.

[5] A. Chao, Physics of Collective Beam Instabilities in High Energy Accelerators, John Wiley \& Sons, 1993.

[6] R. L. Gluckstern, J. van Zeijts, and B. Zotter, "Coupling impedance of beam pipes of general cross-section", Phys. Rev. E47 (1993) 656-663.

[7] S. Heifets, A. Wagner, and B. Zotter, "Generalized impedances and wakes in asymmetric structures", SLAC-AP-110, Jan. 1998.

[8] A. Chao, S. Heifets, and B. Zotter, "Tune shifts of bunch trains due to resistive vacuum chambers without circular symmetry", Phys. Rev. ST Accel. Beams 5 (2002) 111001. 